\title{
Stochastic Framework for Evaluating the Effect of Displaced Antenna Elements on DOA Estimation
}

\author{
Veronique Inghelbrecht, Jo Verhaevert, Tanja Van Hecke, Hendrik Rogier, Marc Moeneclaey, and Herwig Bruneel
}

\begin{abstract}
We establish a statistical framework for investigating the influence of correlated random displacements of antenna elements in a uniform circular antenna array (UCA) on the distribution of direction-of-arrival (DOA) estimates. More specifically, we apply a stochastic collocation method for modeling the sparse UCA root-MUSIC-DOA estimates as polynomial expansions of the random displacements. Compared to Monte-Carlo simulations, this approach yields a speedup of about $\mathbf{4 0}$ for the case of a displacement of two antenna elements.
\end{abstract}

Index Terms-Antenna arrays, direction-of-arrival estimation, stochastic collocation method (SCM).

\section{INTRODUCTION}

$\mathbf{U}$ NIFORM circular antenna arrays (UCAs) are attractive configurations for direction-of-arrival (DOA) estimation, as their circular symmetry may be effectively exploited by algorithms such as UCA root-MUSIC and UCA ESPRIT [1]. Yet, mutual coupling effects may significantly deteriorate the accuracy of the DOA estimates. Therefore, adapted algorithms were proposed that include a calibration procedure that accounts for the exact radiation characteristics of all antennas embedded in the array through a phase-mode or spherical-wave expansion of their impedance matrix and direction-dependent open-circuit voltages [2]. Hence, in most algorithms, perfect knowledge of the steering vector is assumed. Some algorithms, such as the rank reduction estimator (RARE), do not require mutual coupling compensation. Instead, they are based on a symmetry property in the array geometry, which may be embedded in the second-order statistics of the observed data (SOS-RARE) [3]. However, most of these algorithms experience a significant performance loss when random antenna position errors break the symmetry property [4].

Unfortunately, random deviations between the actual and the assumed steering vector are unavoidable in practice, whereas array calibration only removes systematic errors. Indeed, most

Manuscript received March 7, 2016; revised April 27, 2016; accepted May 14, 2016. Date of publication May 20, 2016; date of current version February 27, 2017. This work was supported in part by the Inter-University Attraction Poles Program initiated by the Belgian Science Policy Office.

V. Inghelbrecht, M. Moeneclaey, and H. Bruneel are with the Department of Telecommunications and Information Processing, Ghent University, Ghent 9000, Belgium (e-mail: Veronique.Inghelbrecht@UGent.be; mm@telin.ugent.be; hb@telin.ugent.be).

J. Verhaevert, T. Van Hecke, and H. Rogier are with the Department of Information Technology, Faculty of Engineering and Architecture, Ghent University, Ghent 9000, Belgium (e-mail: Jo.Verhaevert@UGent.be; Tanja.Vanhecke@UGent.be; Hendrik.Rogier@UGent.be).

Color versions of one or more of the figures in this letter are available online at http://ieeexplore.ieee.org.

Digital Object Identifier 10.1109/LAWP.2016.2571319 calibration procedures are time-consuming and, therefore, difficult to apply in real-time applications where the antenna positions change randomly with time (e.g., because of wind, etc.). Hence, it is interesting to quantify the effects of uncertainty in the positions of the sensor elements by a stochastic framework. In [5], a closed-form formula for the mean square error is derived for the FOC-RARE algorithm in the presence of sensor errors based on a first-order perturbation. The algorithm is only valid for sufficiently small displacements of the antenna elements. In [6], we introduced the stochastic collocation method (SCM) [7] to calculate the probability density functions (pdfs) of the DOA estimates due to a random displacement of one sensor element in an acoustical array without mutual coupling. This procedure proved to be much more efficient than the time-consuming Monte-Carlo (M-C) method.

In this letter, we extend the SCM-DOA technique to random displacements of multiple antenna elements in an electromagnetic array with mutual coupling. The random variables describing the position errors are assumed to be multivariate Gaussian distributed and may be correlated. The procedure is illustrated by relying on the Sparse UCA Root-MUSIC [8] DOA estimation algorithm. This method compensates for mutual coupling by incorporating all relevant phase modes of the active elements patterns of the antenna elements in the nominal UCA, even when the number of relevant phase modes is larger than the number of elements. A comparison between the M-C method and the SCM technique shows a reduction in CPU time by a factor of about 40, for the same accuracy in the statistical distribution of the DOA estimates, when two antenna elements are randomly displaced.

Section II describes how we adapt the DOA estimation algorithm for the case of displacements of antenna elements. Section III explains the SCM theory, whereas Section IV summarizes the sparse UCA Root-MUSIC algorithm. Finally, Section V validates the accuracy of the SCM approach.

\section{SignAl MOdEL INCORPORATING ANTENNA DISPLACEMENTS}

The nominal UCA consists of $N$ identical antenna elements distributed over a circle with radius $R$. Each antenna element is located in the $x y$-plane, at azimuth angles $\varphi_{n}=(n-1) \frac{2 \pi}{N}$ with $n=1,2, \ldots, N$. The number of incoming plane waves equals $K$, with corresponding elevation and azimuth angles $\left(\theta_{k}, \varphi_{k}\right), k=1, \ldots, K$. In the following, we point out how to model the received signals in the presence of antenna element displacements, for given $\left(\theta_{k}, \varphi_{k}\right), k=1, \ldots, K$. 
When antenna elements are displaced, both the radiation pattern [9] and the impedance matrix change. The element-space manifold vectors depend on both characteristics and, hence, are affected by a displacement of the antenna elements. In order to account for the antenna element displacements, we adjust the received signals $\mathbf{x}$, as follows.

1) Obtain the $N \times N$ impedance matrix $\mathbf{Z}$ and embedded element patterns $\mathbf{F}_{n}(\theta, \varphi)$ of the actual UCA (being nonsymmetric due to the displacements) for the $K$ DOAs, using an electromagnetic field solver such as NEC-2 [10]. We obtain $\mathbf{F}_{n}(\theta, \varphi)$ [Ohm] (two-dimensional field vector in $\left(\mathbf{u}_{\theta}, \mathbf{u}_{\varphi}\right)$-plane) by exciting antenna $n$ with 1 -A current and leaving the other antennas open-circuited.

2) Calculate [2] the element space manifold vector $\mathbf{a}_{\text {displ }}(\theta, \varphi)$ by

$$
\begin{aligned}
a_{\text {open }, n}(\theta, \varphi) & =-\frac{2 j \lambda}{R_{c}} \mathbf{e}^{\text {inc }}(\theta, \varphi) \cdot \mathbf{F}_{n}(\theta, \varphi) \\
\mathbf{a}_{\text {displ }}(\theta, \varphi) & =Z_{0}\left(\mathbf{Z}+Z_{0} \mathbf{I}\right)^{-1} \mathbf{a}_{\text {open }}(\theta, \varphi)
\end{aligned}
$$

with $\mathbf{e}^{\text {inc }}(\theta, \varphi)[\mathrm{V} / \mathrm{m}]$, a two-dimensional electric field vector of an incoming plane wave at angles $(\theta, \varphi), \lambda$ the wavelength, and $R_{c}$ the impedance of the medium in which the antenna radiates. $Z_{0}$ is the load impedance, which we assume identical at all antennas terminals. The array signal model includes all electromagnetic details, such as mutual coupling effects, through the impedance matrix $\mathbf{Z}$ and the open-circuit voltage vector $\mathbf{a}_{\text {open }}(\theta, \varphi)=$ $\left[a_{1}(\theta, \varphi), \ldots, a_{N}(\theta, \varphi)\right]$.

3) Determine the actual element-space manifold matrix $\mathbf{A}_{\text {displ }}$ and the corresponding received signal vector at instant $t$ as

$$
\mathbf{x}(t)=\mathbf{A}_{\text {displ }} \mathbf{s}(t)+\mathbf{v}(t), \quad t=0,1,2, \ldots
$$

where $\mathbf{s}(t) \in \mathbb{R}^{K \times 1}$ is the transmitted signal vector at time instant $t, \mathbf{x}(t) \in \mathbb{C}^{N \times 1}$ is the received signal vector, and $\mathbf{v} \in \mathbb{C}^{N \times 1}$ is assumed to be a spatially uncorrelated additive white Gaussian noise vector. The $k$ th column of the $(N \times K)$ matrix $\mathbf{A}_{\text {displ }}$ equals the element-space manifold vector $\mathbf{a}_{\text {displ }}\left(\theta_{k}, \varphi_{k}\right)$, determined by the DOA $\left(\theta_{k}, \varphi_{k}\right)$ of the $k$ th plane wave.

Apply the DOA estimation algorithm (in this letter sparse UCA Root-MUSIC, Section IV) to the actual received signal $\mathrm{x}$, assuming the nominal antenna positions, hence a perfect UCA configuration.

\section{Stochastic Collocation Method}

In order to investigate the influence of a random displacement of the antenna elements on the statistical distribution of the DOA estimates, one could resort to M-C simulation. Because of the typically $1 / \sqrt{N_{r}}$ convergence of M-C simulations, a large amount of realizations of the antenna element displacements $N_{r}$ is required to accurately capture the statistics of the DOA estimates. Instead, we apply the SCM, proposed in [6] and [7], as a more efficient way to model geometrical uncertainty. Here, we extend the method from [6], which considers the displacement of only one of $N$ antenna elements of a uniform linear array, to multiple correlated Gaussian displacements; the number of displacements is denoted $O$.
First, we apply a Karhunen-Loève transform [11] to decorrelate the Gaussian random variables: The vector $\mathbf{u}$ of $O$ correlated displacements with covariance matrix $\boldsymbol{\Sigma}$ is related to a vector $\boldsymbol{\eta}$ of $O$ statistically independent variables through $\mathbf{u}=\sqrt{2} \mathbf{V} \sqrt{\boldsymbol{\Lambda}} \boldsymbol{\eta}$, where the columns of $\mathbf{V}$ are the eigenvectors of $\boldsymbol{\Sigma}$, and $\boldsymbol{\Lambda}$ is the diagonal matrix containing the corresponding eigenvalues. The pdf of $\boldsymbol{\eta}$ is given by $p(\boldsymbol{\eta})=$ $(2 / \pi)^{L / 2} \exp \left(-|\boldsymbol{\eta}|^{2}\right)$. The $K$ DOA estimates, which result from the actual nonsymmetric UCA, are functions of the $O$ independent variables $\boldsymbol{\eta}$, which are related to the displacement vector u. Stacking the DOA estimates into the $K \times 1$ vector $\mathbf{y}(\mathbf{u})$, we rely on the Askey scheme [12] to approximate $\mathbf{y}(\sqrt{2} \mathbf{V} \sqrt{\Lambda} \eta)$ as a truncated polynomial expansion of the variables $\boldsymbol{\eta}$, i.e., $\mathbf{y}(\sqrt{2} \mathbf{V} \sqrt{\Lambda} \boldsymbol{\eta}) \approx \mathbf{f}(\sqrt{2} \mathbf{V} \sqrt{\Lambda} \boldsymbol{\eta})$, with

$$
\mathbf{f}(\sqrt{2} \mathbf{V} \sqrt{\boldsymbol{\Lambda}} \boldsymbol{\eta})=\sum_{i_{1}, \ldots, i_{O}} \mathbf{y}_{i_{1}, \ldots, i_{O}} H_{i_{1}}\left(\eta_{1}\right) \ldots H_{i_{O}}\left(\eta_{O}\right)
$$

where $H_{i}(v)$ represents the Hermite polynonial of degree $i$, and the summation index $i_{l}$ ranges from 0 to $P_{l}(l=$ $1, \ldots, O)$; hence, the expansion (4) has a degree $P_{\text {tot }}=P_{1}+$ $\cdots+P_{O}$. Considering that the Hermite polynomials satisfy $\int H_{i}(v) H_{j}(v) e^{-v^{2}} d v=\delta_{i, j}$ with $\delta_{i, j}$ representing the Kronecker delta function, it follows that the mean-square approximation error $\int|\mathbf{y}(\sqrt{2} \mathbf{V} \sqrt{\boldsymbol{\Lambda}} \boldsymbol{\eta})-\mathbf{f}(\sqrt{2} \mathbf{V} \sqrt{\boldsymbol{\Lambda}} \boldsymbol{\eta})|^{2} p(\boldsymbol{\eta}) \mathrm{d} \boldsymbol{\eta}$ is minimized when the coefficients $\mathbf{y}_{i_{1}, \ldots, i_{O}}$ of the polynomial expansion (4) are given by

$$
\mathbf{y}_{i_{1}, \ldots, i_{O}}=\int \mathbf{y}(\sqrt{2} \mathbf{V} \sqrt{\boldsymbol{\Lambda}} \boldsymbol{\eta}) H_{i_{1}}\left(\eta_{1}\right) \ldots H_{i_{O}}\left(\eta_{O}\right) p(\boldsymbol{\eta}) \mathrm{d} \boldsymbol{\eta} .
$$

We approximate the integral (5) by cubature formulas with weight function $e^{-|\boldsymbol{\eta}|^{2}}$ [13], yielding

$$
\mathbf{y}_{i_{1}, \ldots, i_{O}}=\sum_{k=1}^{Q} w_{k} \mathbf{y}\left(\sqrt{2} \mathbf{V} \sqrt{\boldsymbol{\Lambda}} \boldsymbol{\eta}_{k}\right) H_{i_{1}}\left(\eta_{k, 1}\right) \ldots H_{i_{O}}\left(\eta_{k, O}\right)
$$

with $\boldsymbol{\eta}_{k}=\left[\eta_{k, 1}, \ldots\right]$ the quadrature points and $w_{k}$ the weights associated with $\boldsymbol{\eta}_{k}$. The degree $d_{e}$ of the cubature formula denotes that (6) is exact for polynomials of degree up to $d_{e}$. The cubature formulas applied in this letter are those from [13] with $\left(Q=9, d_{e}=5\right),\left(Q=44, d_{e}=15\right)$, and $\left(Q=99, d_{e}=21\right)$. The maximum degree of expansion must satisfy $P_{\text {tot }} \leq d_{e} / 2$.

The computation of the coefficients $\mathbf{y}_{i_{1}, \ldots, i_{O}}$ according to (6) requires the actual element-space manifold matrix $\mathbf{A}_{\text {displ }}$ computation [to be used in (3)] and the subsequent DOA estimation [based on $\mathbf{x}(t)$ from (3)] to be executed for only $Q$ sets of displacements corresponding to the $Q$ quadrature points. An arbitrary number $N_{r}$ of realizations of the DOA estimate vector, each corresponding to a different displacement vector $\mathbf{u}$, is obtained by simply evaluating (4) for $N_{r}$ realizations of $\boldsymbol{\eta}$. An $\mathrm{M}-\mathrm{C}$ approach would require the actual element-space manifold matrix $\mathbf{A}_{\text {displ }}$ and the corresponding DOA estimates to be computed for each of the $N_{r}$ realizations of $\mathbf{u}$. Hence, considering the high computational complexity of the actual element-space manifold matrix $\mathbf{A}_{\text {displ }}$, the SCM has a considerable computational advantage over the M-C approach when $N_{r} \gg Q$, which will be more important for even more complex antenna arrays. 


\section{SPARSE UCA RoOT-MUSIC AlgORITHM}

We now provide a brief summary of the sparse UCA rootMUSIC algorithm [8]. We decompose the component $a(\theta, \varphi)$ into phase modes [14], which can be efficiently evaluated as a fast Fourier transform

$$
a(\theta, \varphi)=\sum_{m=-\infty}^{+\infty} a_{m}(\theta) e^{j m \varphi} .
$$

The electromagnetic dimensions of the UCA restrict the number of relevant terms in (7) to $2 M+1$, i.e., $|m| \leq$ $M$, with $M \geq \frac{2 \pi R}{\lambda}$ [8]. The weight vector that excites the array with the $m$ th phase mode is given by $\mathbf{w}_{m}^{H}=$ $1 / \sqrt{N}\left[1, e^{j 2 m \pi / N}, \ldots, e^{j 2 m(N-1) \pi / N}\right]$

$$
\mathbf{w}_{m}^{H} \mathbf{a}(\theta, \varphi)=a_{m}(\theta) e^{j m \varphi}+\delta(\theta, \varphi)
$$

where

$$
\delta(\theta, \varphi)=\sum_{\substack{q=-\infty \\ q \neq 0}}^{+\infty} a_{m+q N}(\theta) e^{j(m+q N) \varphi}
$$

and the superscript ${ }^{H}$ denotes the transpose conjugate. For $N>$ $2 M$, the first term in (8) is dominant and we can neglect the residual term $\delta(\theta, \varphi)$. When the number of antenna elements is too small $(N=2 L+1$ and $L<M)$, the phase modes of orders $L<|m+q N| \leq M$ contribute to the residual term. A UCA with less than $2 M+1$ antenna elements is denoted as a sparse UCA. Then, the transformation into beamspace is governed by only $2 L+1$ weight vectors: $\mathbf{W}=\left[\mathbf{w}_{-L}, \ldots, \mathbf{w}_{0}, \ldots, \mathbf{w}_{L}\right]$. To apply the sparse UCA Root-MUSIC algorithm [8], we construct the beamspace data: $\mathbf{x}_{\text {beam }}(\theta, \varphi)=\mathbf{W}^{H} \mathbf{x}$. Next, we evaluate the covariance matrix $\mathbf{R}_{\mathrm{cov}}$ of the beamspace data and decompose $\mathbf{R}_{\text {cov }}$ into a signal and noise subspace

$$
\mathbf{R}_{\text {cov }}=\mathrm{E}\left\{\mathbf{x}_{\text {beam }} \mathbf{x}_{\text {beam }}^{H}\right\}=\mathbf{E}_{S} \boldsymbol{\Lambda}_{S} \mathbf{E}_{S}^{H}+\mathbf{E}_{N} \boldsymbol{\Lambda}_{N} \mathbf{E}_{N}^{H} .
$$

The columns of $\mathbf{E}_{S}$ and $\mathbf{E}_{N}$ denote the signal and noise subspace eigenvectors, and the diagonal matrices $\boldsymbol{\Lambda}_{S}$ and $\boldsymbol{\Lambda}_{N}$ contain the signal subspace and noise subspace eigenvalues.

To deliver bias-free estimates for any signal-to-noise ratio (SNR) level, we incorporate all $M>L$ relevant phase modes. Therefore, we define

$$
\begin{aligned}
\mathbf{F}(\theta) & =\operatorname{diag}\left[a_{-M}(\theta), \ldots, a_{M}(\theta)\right] \\
\mathbf{a}_{e}(z) & =\left[z^{-M}, \ldots, z^{M}\right] \text { with } z=e^{j \varphi} .
\end{aligned}
$$

Define the matrix $\mathbf{H}$ by

$$
\begin{aligned}
\mathbf{H} & =\left[\mathbf{J}_{l, 1} \vdots \mathbf{I} \vdots \mathbf{J}_{r, 1}\right] \text { if } 2 L+1<M \leq 3 L+1 \\
& =\left[\mathbf{J}_{l, 2} \vdots \mathbf{I} \vdots \mathbf{I} \vdots \mathbf{I} \vdots \mathbf{J}_{r, 2}\right] \text { if } 3 L+1<M \leq 5 L+2
\end{aligned}
$$

with the matrix $\mathbf{I}$ a $(2 L+1) \times(2 L+1)$ identity matrix. $\mathbf{J}_{r, 1}$ is a $(2 L+1) \times(M-L)$ matrix consisting of the $M-L$ first columns of the identity matrix $\mathbf{I}$, and $\mathbf{J}_{r, 2}$ is a $(2 L+$ 1) $\times(M-3 L-1)$ matrix consisting of the $M-3 L-1$ first columns of the identity matrix $\mathbf{I}$. $\mathbf{J}_{l, 1}$ is a $(2 L+1) \times(M-L)$ matrix consisting of the $M-L$ last columns of the identity matrix $\mathbf{I}$, and $\mathbf{J}_{l, 2}$ is a $(2 L+1) \times(M-3 L-1)$ matrix consisting of the $M-3 L-1$ last columns of the identity matrix I. For $M=L$, the sparse UCA Root-MUSIC reduces to the traditional UCA Root-MUSIC. Finally, assuming that the elevation angles of the impinging signals are known, the MUSIC function can be written as

$$
f_{\text {MUSIC }}(z)=\mathbf{a}_{e}^{H}(z) \mathbf{F}^{H}(\theta) \mathbf{H}^{H} \mathbf{E}_{N} \mathbf{E}_{N}^{H} \mathbf{H F}(\theta) \mathbf{a}_{e}(z)
$$

enabling us to estimate the azimuth angles by finding the $K$ roots of the polynomial $f_{\text {MUSIC }}(z)$ closest to the unit circle. The DOA estimates are the arguments of these $K$ roots.

\section{NUMERICAL RESULTS}

Consider a uniform circular array consisting of nine dipole antenna elements tuned to $2.45 \mathrm{GHz}$. (dipole length $l=\lambda / 2=6.12 \mathrm{~cm}$ ). All antennas are terminated by a load $Z_{0}=73 \Omega$. In the center of the circle, there is a short-circuited dipole with length $l=\lambda / 2$. The $N=\left\lceil 8 \frac{R}{\lambda}+4\right\rceil=9$ array elements are uniformly distributed on a circle with radius $\lambda / 2$. The short-circuited dipole acts as a platform effect. The phase modes of the UCA, incorporating all electromagnetic effects and simulated with the 4 nec 2 field simulator, differ significantly from the phase modes of an identical hypothetical UCA consisting of ideal uncoupled dipoles. We consider $K=3$ uncorrelated sources, each transmitting pseudorandom bit sequences of length 500, using BPSK modulation. These signals are impinging on the UCA along the same elevation angle $\theta=90^{\circ}$ and along the azimuth angles $\left[20^{\circ}, 200^{\circ}, 285^{\circ}\right]$. The signals are received in the presence of additive white Gaussian noise $\mathbf{v}(t)$. The SNR (in $\mathrm{dB}$ ) is defined as

$$
\mathrm{SNR}=10 \log _{10}\left(\frac{E\left\{\|\mathbf{A s}(t)\|^{2}\right\}}{E\left\{\|\mathbf{v}(t)\|^{2}\right\}}\right) .
$$

We take SNR $=25 \mathrm{~dB}$. In order to obtain the DOA estimates, we use the sparse UCA Root-MUSIC algorithm, with $L=$ floor $((N-1) / 2)$ and $M=10 \geq \operatorname{ceil}(2 \pi R / \lambda)$. We consider the case where the first and second antenna element have random radial displacements $u_{1}$ and $u_{2}$, while the remaining antenna elements are at their nominal positions. These random variables are assumed to be multivariate Gaussian distributed with zero mean and covariance matrix (expressed in $\mathrm{cm}$ )

$$
\boldsymbol{\Sigma}=\left(\begin{array}{lc}
1 & 0.7 \\
0.7 & 1
\end{array}\right)
$$

To obtain the cumulative distributions of the estimated azimuth angles, we apply two different methods: M-C simulation and the two-dimensional SCM theory. We have carried out an M-C simulation with 10000 realizations of $\left(u_{1}, u_{2}, \mathbf{v}\right)$. For each of these realizations, we estimate the three DOAs, yielding 10000 DOA estimates per actual DOA.

We compare this to the two-dimensional SCM theory that relies on the cubature formulas for the plane with $Q=9, Q=44$, and $Q=99$ quadrature points, and with a polynomial expansion of order $P_{\mathrm{tot}}=2, P_{\mathrm{tot}}=7$, and $P_{\mathrm{tot}}=10$, respectively. In order to incorporate the noise vector $\mathbf{v}$ in the SCM model, we calculate for a random realization of $\mathbf{v}$ the DOA estimates in each of the $Q$ quadrature points $\left(u_{1}, u_{2}\right)$ and determine the coefficients of the polynomial expansion. Using this polynomial expansion, we calculate the DOA estimates for 500 realizations 


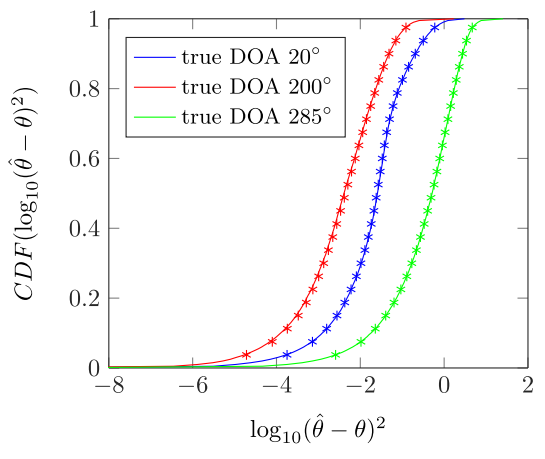

Fig. 1. CDF obtained with M-C (solid line) method and SCM (asterisk) of $\log _{10}(\hat{\theta}-\theta)^{2}$, with $\theta$ and $\hat{\theta}$ in degrees, for a radial displacement of the first two antenna elements, true DOAs $=\left[20^{\circ}, 200^{\circ}, 285^{\circ}\right]$.

TABLE I

Measured Value $D_{n, n^{\prime}}$ OF THe KS Test (Critical Value $=0.0192$ ), DisPlaCEMENT OF FIRST AND SECOND ANTENNA ElEMENT, TRUe DOAS = $\left[20^{\circ}, 200^{\circ}, 285^{\circ}\right]$; THE LAST COLUMN IS THE SimUlation Time To ObTAIN THE 3 CDFs

\begin{tabular}{lccccc}
\hline \hline \multicolumn{5}{c}{$D_{n, n^{\prime}}$ for } \\
\hline$P_{\text {tot }}$ & $Q$ & $\theta_{1}$ & $\theta_{2}$ & $\theta_{3}$ & time \\
\hline 2 & 9 & 0.0232 & 0.0419 & 0.0138 & $51 \mathrm{~s}$ \\
7 & 44 & 0.0189 & 0.0154 & 0.0071 & $100 \mathrm{~s}$ \\
10 & 99 & 0.0098 & 0.0082 & 0.0053 & $205 \mathrm{~s}$ \\
\hline \hline
\end{tabular}

of $\left(u_{1}, u_{2}\right)$, given the considered realization of $\mathbf{v}$. We repeat this procedure for 19 other randomly chosen noise vectors $\mathbf{v}$, and eventually obtain 10000 DOA estimates per actual DOA. For lower SNR, the effect of the noise on the DOA estimation error increases, so we need more realizations of $\mathbf{v}$ to accurately capture the noise statistics.

In Fig. 1, we have plotted the cumulative distribution functions (cdfs) of $\log _{10}(\hat{\theta}-\theta)^{2}$ with $\theta$ and $\hat{\theta}$ in degrees, obtained by the M-C method and by the SCM (for $Q=44$ quadrature points and for the three actual DOAs $\left.\left[20^{\circ}, 200^{\circ}, 285^{\circ}\right]\right)$. We observe that the curves obtained by SCM theory and the curves obtained by $\mathrm{M}-\mathrm{C}$ simulation are virtually identical. However, the simulation time to obtain the three cdfs using M-C simulation is $7870 \mathrm{~s}$, whereas the SCM for 44 quadrature points only requires $100 \mathrm{~s}$. The accuracy of the cdf can be demonstrated by means of the two-sample Kolmogorov-Smirnov test (KS test) [15]. This test verifies the null hypothesis that the two sample sets belong to the same distribution. The test statistic is defined as $D_{n, n^{\prime}}=\sup _{x}\left(\left|F_{1, n}(x)-F_{2, n^{\prime}}(x)\right|\right)$, with $\sup _{x}(f(x))$ the supremum of the function $f(x)$. The functions $F_{1, n}(x)$ and $F_{2, n^{\prime}}(x)$ denote the empirical cdfs obtained with the M-C simulation and with SCM, respectively, where $n$ and $n^{\prime}$ are the number of samples for the two methods. For $n=n^{\prime}=10000$ samples, the two-sample KS test accepts, with a confidence of $95 \%$, that the SCM samples and the M-C samples have the same distribution when $D_{n, n^{\prime}}<0.0192$.

In Table I, we show the values of the test statistic $D_{n, n^{\prime}}$ for different values of the number of quadrature points $Q$. We observe that the null hypothesis is accepted for $Q=44$ and $Q=99$ quadrature points. We also show the time necessary to obtain the three cdfs using the SCM method in the last column, which is substantially less than the $7870 \mathrm{~s}$ for M-C simulation in all cases.

\section{CONCLUSION AND FUTURE WORK}

For a UCA with a shorted-circuited dipole in its center, we have investigated the influence of correlated Gaussian random radial displacements of two out of nine antenna elements to the DOA estimates. We have applied the SCM formalism, using cubature formulas to approximate the DOA estimates as a polynomial expansion of the antenna element displacements. Comparison of the SCM to the M-C method demonstrates that the SCM method accurately predicts the CDFs of the DOAS, for a reduction in CPU time of about 40.

However, with this approach, the number of function evaluations increases exponentially with the number of random variables. In future work, we will study other techniques [16] to investigate the influence on DOA estimates of multiple threedimensional displacements of antenna elements.

\section{REFERENCES}

[1] C. Mathews and M. Zoltowski, "Eigenstructure techniques for 2-D angle estimation with uniform circular arrays," IEEE Trans. Signal Process., vol. 42, no. 9, pp. 2395-2407, Sep. 1994.

[2] R. Goossens and H. Rogier, "A hybrid UCA-RARE/root-music approach for 2-D direction of arrival estimation in uniform circular arrays in the presence of mutual coupling," IEEE Trans. Antennas Propag., vol. 55, no. 3, pp. 841-849, Mar. 2007.

[3] C. Qi, Y. Wang, Y. Zhang, and H. Chen, "DOA estimation and selfcalibration algorithm for uniform circular array," Electron. Lett., vol. 41, no. 20, pp. 1092-1094, Sep. 2005.

[4] M. Viberg, M. Lanne, and A. Lundgren, Calibration in Array Processing, T. Tuncer and B. Friedler, Eds. San Francisco, CA, USA: Academic, 2009.

[5] D. Wang, "Performance of cumulant-based rank reduction estimator in presence of unexpected modeling errors," J. Central South Univ., vol. 22, no. 3, pp. 992-1001, Mar. 2015.

[6] V. Inghelbrecht, J. Verhaevert, T. Van Hecke, and H. Rogier, "The influence of random element displacement on DOA estimates obtained with (KhatriRao-)root-MUSIC," Sensors, vol. 14, pp. 21258-21280, Nov. 2014.

[7] H. Rogier and F. Boeykens, "An efficient technique based on polynomial chaos to model the uncertainty in resonance frequency of textile antennas due to bending," IEEE Trans. Antennas Propag., vol. 62, no. 3, pp. 1253-1260, Mar. 2014.

[8] R. Goossens and H. Rogier, "UCA Root-MUSIC with sparse uniform circular arrays," IEEE Trans. Signal Process., vol. 56, no. 8, pp. 4095 4099, Aug. 2008.

[9] C. A. Balanis, Antenna Theory Analysis and Design. Hoboken, NJ, USA: Wiley, 2005

[10] A. Voors. NEC based antenna modeler and optimizer. (May 2012). [Online]. Available: http://www.qsl.net/4nec2/

[11] T. El-Moselhy, "Field solver technologies for variation-aware interconnect parasitic extraction," Ph.D. dissertation, Dept. of Elect. Eng. Comput. Sci., Massachusetts Inst. of Technol., Cambridge, MA, USA, 2005.

[12] D. Xiu, "Fast numerical methods for stochastic computations: A review," Commun. Comput. Phys., vol. 5, nos. 2-4, pp. 242-272, Feb. 2009.

[13] R. Cools, L. Pluym, and D. Laurie, "Cubpack++: A C++ package for automatic two-dimensional cubature," ACM Trans. Math. Softw., vol. 23, no. 1, pp. 1-15, Mar. 1997.

[14] H. Rogier and E. Bonek, "Analytical spherical-mode based compensation of mutual coupling in uniform circular arrays for directionof-arrival estimation," Int. J. Elect. Commun., vol. 60, pp. 179-189, Mar. 2006.

[15] G. Marsaglia, W. Tsang, and J. Wang, "Evaluating Kolmogorov's distribution," J. Stat. Softw., vol. 8, no. 18, pp. 1-4, Nov. 2003.

[16] P. Manfredi, D. Van de Ginste, D. De Zutter, and F. G. Canavero, "Generalized decoupled polynomial chaos for nonlinear circuits with many random parameters," IEEE Microw. Wireless Compon. Lett., vol. 25, no. 8, pp. 505-507, Aug. 2015. 